\title{
Moistening Deformation and Microstructure Change of Undisturbed Loess Under Confined Conditions
}

\author{
Ying Gao, Yanxia Ma*, Wuyu Zhang, Jiaqing Guo \\ School of Civil Engineering, Qinghai University, Xining, China \\ Email address: \\ yunrunlian@163.com (Ying Gao), myxdyp@163.com (Yanxia Ma) \\ ${ }^{*}$ Corresponding author
}

To cite this article:

Ying Gao, Yanxia Ma, Wuyu Zhang, Jiaqing Guo. Moistening Deformation and Microstructure Change of Undisturbed Loess Under Confined Conditions. International Journal of Environmental Protection and Policy. Vol. 7, No. 2, 2019, pp. 80-85.

doi: $10.11648 /$ j.ijepp.20190702.15

Received: April 22, 2019; Accepted: May 23, 2019; Published: June 15, 2019

\begin{abstract}
Under the confined conditions, the influence of water content on the deformation characteristics of loess collapsing was studied by the indoor collapsibility test. The microscopic images were analyzed microstructural changes of the soil before and after the collapsing. The result shows that: (1) the higher the moisture content, the smaller vertical deformation of the sample after immersion in water under the same immersion pressure, and the smaller collapsibility. On the contrary, when the moisture water content is small, the main form of deformation is water immersion. After the collapse deformation, the amount of compression will decrease; (2) with the increase of water content, under the joint action of pressure and water, the soil structure tends to be dense, the particle filling area becomes larger, and the pore filling area decreases, and the pores are filled with clay and fine particles, and the connectivity between pores is reduced; (3) the average pore diameter, average pore area and average pore circumference of different soil samples after immersion are gradually reduced compared with the soil sample before immersion. The area is evenly distributed in each angular region and is related to macroscopic collapse deformation.
\end{abstract}

Keywords: Collapsible Loess, Humidification, Pore Structure

\section{Introduction}

The most important mechanical property of loess is its collapsibility, because the loess with natural structure and strong structure. It is often under-pressure-tight state, and its elastic deformation is very small, mainly reason for compaction and deformation. The compact deformation is manifested as compression deformation and collapse deformation. The collapsible deformation develops rapidly after immersion in water, and it has great harm to buildings. It is the main problem facing the construction of loess area. In this regard, domestic and foreign research on this aspect has achieved a lot of results. Through the confined compression test, the researchers proposed the structural damage ratio of loess under the condition of considering the coupling of loading and humidification, and established the settlement checking method of collapsible loess foundation based on structural damage evolution [1-2], the original $\mathrm{Q}_{3}$ the loess adopts the two-wire method to carry out the uniaxial compression test of increasing and decreasing humidity. The research shows that the curve of the collapsibility coefficient of undisturbed loess [3-4], the compression test of Xi'an and Lanzhou undisturbed loess under different water contents is proposed. The calculation method of humidification and collapsibility coefficient is studied, and the relationship between humidification collapsibility coefficient and water content under the same pressure is studied [5]. The researchers used the indoor compression test to investigate compressive deformation and collapsible deformation characteristics of loess during the process of humidification. It was found that the compressibility of loess increased with the increase of water content; the collapsibility was weakened. As the overburden pressure increases, the collapsibility is greater. And when the overburden pressure is smaller, the humidification and collapsibility are weaker. Compressive deformation and collapsible deformation can be converted into each other during humidification [6-10]. With the help of electronic scanning microscopy, the scholars found that the 
water content of Yili loess is small, and the contact between the particles is mainly in the form of point contact and overhead structure. These factors are the historical reasons and internal causes of the strong collapsibility, and the effect of the overhead pores is greatly affected. It is sensitive to the action of water. During the process of collapsing, the large pores become medium and small pores, and the small and medium pores become smaller pores [11-15].

The soil in the actual foundation is usually confined, so it should be studied under the confinement conditions during the test. In this paper, the soil sample with natural water content is pre-wet method to obtain soil samples with different moisture water content by using conventional compression instrument. Then the soil samples under different humidification water content are saturated with water under water immersion pressure to study the original loess collapsibility. The influence of the deformation characteristics combined with the microstructure of the loess is analyzed by the loess deformation characteristics exhibited by the loess.

\section{Test Plan and Method}

\subsection{Test Soil}

The test soil was taken from a construction site in Xining City, and the artificial exploration well was used to take the original soil sample. When sampling, remove the miscellaneous soil on the surface layer of the site, the sampling depth is $4 \mathrm{~m}$, wrap the sample taken with plastic wrap, install the plastic bag and seal with tape to prevent water loss. In addition, the sealed soil samples are wrapped and packed in a cushion film to prevent the soil samples from being damaged by collision or vibration during transportation.

Table 1. Physical properties of loess.

\begin{tabular}{|c|c|c|c|c|c|c|c|c|}
\hline \multirow{2}{*}{$\begin{array}{l}\text { Depth of } \\
\text { soil } / \mathbf{m}\end{array}$} & \multirow{2}{*}{$\begin{array}{l}\text { Natural moisture } \\
\text { content } / \%\end{array}$} & \multirow{2}{*}{$\begin{array}{l}\text { Soil specific } \\
\text { gravity }\end{array}$} & \multirow{2}{*}{$\begin{array}{l}\text { Liquid limit } \\
/ \%\end{array}$} & \multirow{2}{*}{$\begin{array}{l}\text { Plastic } \\
\text { limit/\% }\end{array}$} & \multirow{2}{*}{$\begin{array}{l}\text { Plasticity } \\
\text { index }\end{array}$} & \multirow{2}{*}{$\begin{array}{l}\text { Dry density } \\
\mathrm{g} / \mathrm{cm}^{3}\end{array}$} & \multicolumn{2}{|c|}{ Particle composition /\% } \\
\hline & & & & & & & $0.075 \sim 0.005 \mathrm{~mm}$ & $<0.005 \mathrm{~mm}$ \\
\hline 4 & 10.5 & 2.71 & 28.67 & 13.56 & 15.11 & 1.44 & 88.3 & 11.7 \\
\hline
\end{tabular}

\subsection{Collapsibility Test}

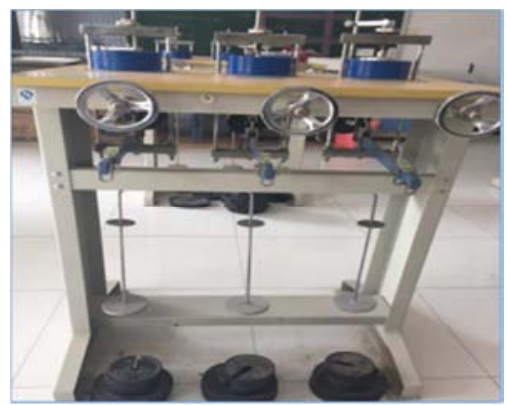

Figure 1. WG type single lever consolidation instrument.

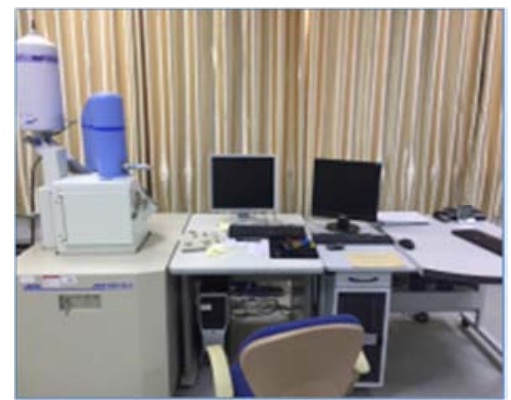

Figure 2. JSM-6610LV type electron microscope.

The test instrument is a WG type single-lever consolidation instrument, which adopts the two-wire method and the single-wire method. The sample size is $50 \mathrm{~cm}^{2}$ and the height is $20 \mathrm{~mm}$. The test pressures are $12.5 \mathrm{kPa}, 25 \mathrm{kPa}, 50 \mathrm{kPa}, 100$ $\mathrm{kPa}, 150 \mathrm{kPa}, 200 \mathrm{kPa}, 300 \mathrm{kPa}, 400 \mathrm{kPa}$. The water content is $7 \%, 12 \%, 17 \%, 22 \%$, and the soil sample is humidified to different water content. The method is prepared by titration and water or air drying. The two ends of the water content are $20 \mathrm{~mm}$ higher than the two ends. The ring knife sample is placed in a closed moisturizing tank for about 3 days. The soil sample at both ends of the cutting ring cutter is tested to determine whether the moisture content reaches the required moisture content, thereby ensuring the uniformity of moisture content in the sample. Using the two-wire method, one sample is graded and loaded under natural humidity, and the other sample is saturated with water. After the sinking is stabilized, the two samples are simultaneously added to the final stage pressure, and the standard for sinking deformation is $0.01 \mathrm{~mm} / \mathrm{h}$.

\subsection{Microscopic Test}

This test instrument (shown in Figure 2) JSM-6610LV electron microscope quantitatively analyzes the relationship between macroscopic collapsible deformation and compressive deformation of loess and microstructure characteristics before and after water immersion in different water contents.

The JSM-6610LV electron microscope was used to quantitatively analyze the relationship between the macroscopic collapse deformation and compression deformation of the loess and the microstructure characteristics.

(1) Preparation of microscopic sample: A microscopic scanning sample having a size of $1 \mathrm{~cm} \times 1 \mathrm{~cm} \times 2 \mathrm{~cm}$ (length $\times$ width $\times$ height) was prepared, and a groove of about $1 \mathrm{~mm}$ deep was engraved with a small knife in the middle of the long side to facilitate the preparation of a natural section. Before the microscopic scanning with an electron microscope, it is necessary to carry out two steps of drying and spraying the sample.

(2) Microscopic sample image acquisition: Microscopic samples were placed under 300 and 800 times scanning electron microscopy to collect microscopic images before and after water immersion in soils with different humidification 
moisture content. Observe with SEM, move the soil sample within the line of sight of the lens, remove some special points, and find some representative parts to take pictures.

(3) Microscopic image processing: The SEM image is subjected to parameter calculation and parameter classification of digital image under the Particles and cracks analysis system (PCAS) program. The data extraction and analysis of the loess microstructure image is compared with the average shape factor and distribution fractal dimension. Pore area ratio and other parameters.

\section{Analysis of Test Results}

\subsection{Characteristics of Loess Collapsibility Curve Under Different Moisture Water Content}

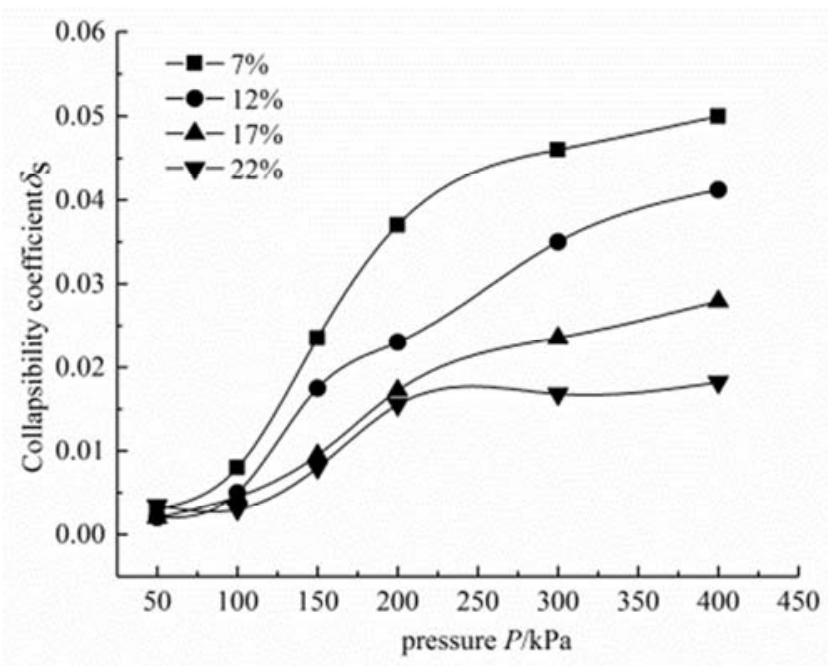

Figure 3. The coefficient of collapsibility coefficient of different initial water content.

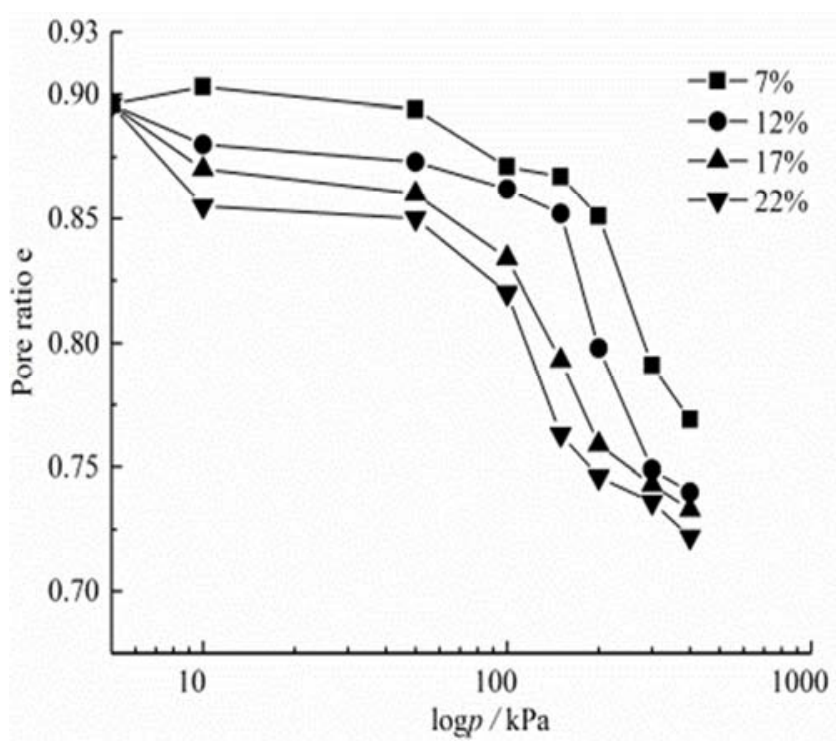

Figure 4. The e-logP curve of different initial water content.

It can be seen from figure 3 and figure 4 that for the undisturbed loess, the collapsibility coefficient $\delta$ s decreases with the increase of initial water content, and the $\mathrm{P}-\delta \mathrm{s}$ relationship curve decreases gradually, and $\mathrm{P}-\delta$ s tends to be close when the initial water content is close to saturation. It is gentle, indicating that the collapsibility of undisturbed loess decreases with humidification. Because the initial water content determines the proportional relationship between the compression deformation and the collapse deformation of the soil, when the initial water content is low, the soil has a small compression deformation under a certain pressure, and the collapse deformation is large. Otherwise, when the initial water content is high, the compression deformation is large and collapse deformation is small; the lower moisture water content, the greater vertical deformation of the sample after water immersion. And under the same immersion pressure, the greater collapsibility, the soil moisture determines the soil due to the initial water content. The proportional relationship between compressive deformation and collapsible deformation of the body. When the initial water content is low, the main form of deformation of the soil under a certain pressure is the collapse deformation after immersion, and when the humidification water content is high, the main form of compression deformation before immersion, the amount of collapsibility will decrease. When the collapsibility coefficient $\delta \mathrm{s}$ reaches 0.015 , the corresponding minimum collapse pressure is defined as the initial collapse pressure of loess, which marks the beginning of loess collapsibility; the maximum collapsible pressure when the collapsibility coefficient is greater than or equal to 0.015 is defined as loess. The collapse end pressure indicates the end of the loess collapse. It can be seen from the figure that the initial collapse pressure of undisturbed loess increases with the initial water content, and the collapse end pressure decreases with the increase of initial water content. Under the same moisture water content, the greater immersion pressure, the greater deformation of the sample after immersion in water saturation, and the greater collapsibility. The moisture content of loess has an important influence on its collapsibility. In general, with the increase of water content, the collapsibility of loess is reduced, and variation of the collapsible amount is different in different water content intervals.

\subsection{Microstructure Changes of Loess Under Different Moisture Water Content}

In the study of the soil microstructure before and after the collapse or compression, considering the small load pressure applied in this test, the influence on the individual particles in the structure is small, while the particle alignment and pore structure characteristics have more obvious influence, through the analysis of SEM images. This paper attempts to study the variation characteristics of collapsible loess from external factors from the perspective of microstructure, and provides a new way to explore the collapsible deformation and compression deformation of loess.

\subsubsection{Qualitative Analysis of Loess Microstructure Under Different Moisture Water Content}

A comparative analysis of the microstructure image with magnification of 800 times (figure 5 - figure 8) shows the 
particle arrangement and pore distribution. The particle unit is relatively loosely bound, the particle size is different, and small particles gather around the large particles. The observable pore system is also relatively complete, and the shape and size of the intergranular pores in the granule and the granulating unit can be clearly distinguished, and the large pore structure is more remarkable. With the increase of water content, the microscopic image of the sample before immersion shows that the soil structure changes from loose to dense after the original loess is compressed. The particle unit is arranged more closely, and the number of pores decreases accordingly, but the joint structure, It still exists between the granular unit bodies. The original loose particles are more densely packed after compression, and the number of pores is reduced as a whole, and the shape regularity tends to be regular. With the increase of water content, the microscopic image of the sample after water immersion shows that under the joint action of pressure and water, the soil structure tends to be dense, the particle filling area becomes larger, and the pore filling area decreases. The collapsible process of loess is characterized by the transition from unstable stent contact to stable inlaid contact between the framework particles. A large amount of fine particles are adhered to the surface of the particles. The number of large and medium pores is obviously reduced, the number of small and tiny pores is increased, and the pores are filled. Cohesive and fine-grained materials, the connectivity between pores is reduced.
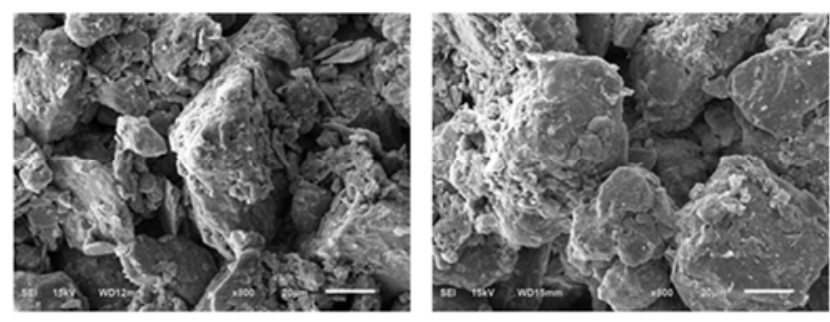

Figure 5. $w=7 \%$ before and after water immersion microstructure.
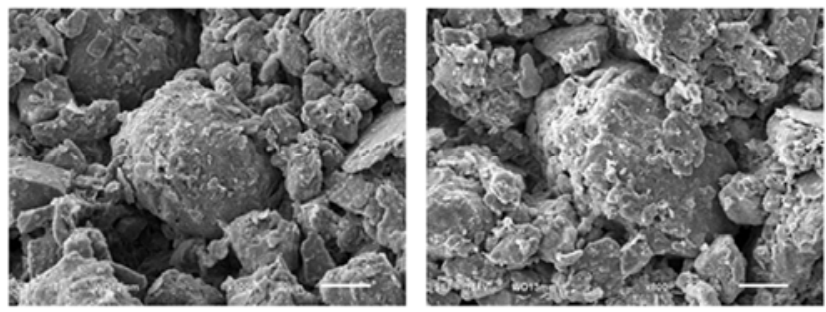
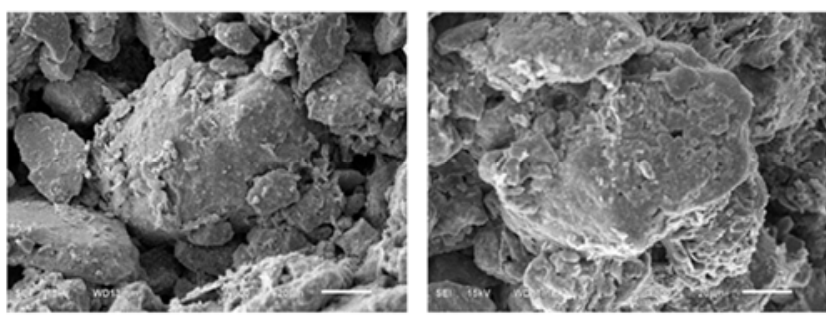

Figure 7. $w=17 \%$ before and after water immersion microstructure.
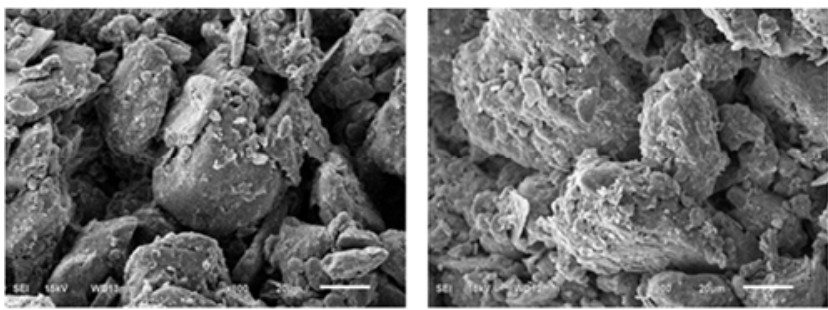

Figure 8. $w=22 \%$ before and after water mmersion microstructure.

\subsubsection{Quantitative Analysis of the Microstructure of Loess Under Different Humidified Water Contents}

According to Lei Xiang yi's classification scheme, there are: the pore size of the large pores is $r \geq 25 \mu \mathrm{m}$, the large pores are $\mathrm{r}=25 \sim 16 \mu \mathrm{m}$, the aperture $\mathrm{r}=16 \sim 4 \mu \mathrm{m}$, the aperture $\mathrm{r}=4 \sim$ $1 \mu \mathrm{m}$, and the aperture $\mathrm{r}<1 \mu \mathrm{m}$. The pores of different pore sizes after immersion in water with different water contents were counted. The results are shown in Table 2 and Table 3.

It can be seen from Table 2 that the average pore diameter, average pore area and average pore circumference of different water contents before and after water immersion gradually decrease with the increase of water content, and the original aperture structure is damaged to varying degrees. After immersion, the soil structure system is more encrypted. The number and area of pores are relatively reduced, and the average shape factor increases with the increase of water content. The circularity of the particles after soil immersion has a tendency to decrease gently with respect to the circular shape of the pores before immersion. It can be seen from Table 3 that with the increase of water content, under the joint action of pressure and water, the content of large and medium pores after immersion is gradually reduced compared with that before immersion, and the content of micro and small pores increases, indicating that loess is wet. After the trapped soil, the pores are reduced and the grain structure is gradually dense.

Figure 6. $w=12 \%$ before and after water immersion microstructure.

Table 2. Pore characteristics of different water content.

\begin{tabular}{|c|c|c|c|c|c|c|c|c|}
\hline \multirow{2}{*}{$\begin{array}{l}\text { Water } \\
\text { content } \\
\%\end{array}$} & \multicolumn{2}{|c|}{ Average pore diameter $/ \mu \mathrm{m}$} & \multicolumn{2}{|c|}{ Average pore area $/ \mu \mathrm{m}^{2}$} & \multicolumn{2}{|c|}{ Average porosity Perimeter/ $\mu \mathrm{m}$} & \multicolumn{2}{|c|}{ Average shape factor } \\
\hline & $\begin{array}{l}\text { Before } \\
\text { immersion }\end{array}$ & $\begin{array}{l}\text { After } \\
\text { immersion }\end{array}$ & $\begin{array}{l}\text { Before } \\
\text { immersion }\end{array}$ & $\begin{array}{l}\text { After } \\
\text { immersion }\end{array}$ & Before immersion & $\begin{array}{l}\text { After } \\
\text { immersion }\end{array}$ & $\begin{array}{l}\text { Before } \\
\text { immersion }\end{array}$ & $\begin{array}{l}\text { After } \\
\text { immersion }\end{array}$ \\
\hline $7 \%$ & 8.236 & 6.912 & 33.452 & 31.256 & 36.247 & 35.785 & 0.364 & 0.335 \\
\hline $12 \%$ & 7.558 & 6.756 & 29.584 & 28.654 & 34.215 & 32.524 & 0.373 & 0.319 \\
\hline $17 \%$ & 6.957 & 6.395 & 27.369 & 22.378 & 31.983 & 29.395 & 0.321 & 0.303 \\
\hline $22 \%$ & 6.803 & 6.201 & 23.452 & 21.215 & 28.361 & 26.502 & 0.334 & 0.329 \\
\hline
\end{tabular}


Table 3. Relationship between pore composition and water content.

\begin{tabular}{|c|c|c|c|c|c|c|c|c|}
\hline \multirow{3}{*}{ Water content $\%$} & \multicolumn{8}{|c|}{ Pore content of different pore sizes /\% } \\
\hline & \multicolumn{2}{|l|}{$<1(\mu \mathrm{m})$} & \multicolumn{2}{|l|}{$1 \sim 4(\mu \mathrm{m})$} & \multicolumn{2}{|l|}{$4 \sim 16(\mu \mathrm{m})$} & \multicolumn{2}{|l|}{$\geq 16(\mu \mathrm{m})$} \\
\hline & $\begin{array}{l}\text { Before } \\
\text { immersion }\end{array}$ & $\begin{array}{l}\text { After } \\
\text { immersion }\end{array}$ & $\begin{array}{l}\text { Before } \\
\text { immersion }\end{array}$ & $\begin{array}{l}\text { After } \\
\text { immersion }\end{array}$ & $\begin{array}{l}\text { Before } \\
\text { immersion }\end{array}$ & $\begin{array}{l}\text { After } \\
\text { immersion }\end{array}$ & $\begin{array}{l}\text { Before } \\
\text { immersion }\end{array}$ & $\begin{array}{l}\text { After } \\
\text { immersion }\end{array}$ \\
\hline $7 \%$ & 5.9 & 6.7 & 46.5 & 55.3 & 42.3 & 34.5 & 5.3 & 3.5 \\
\hline $12 \%$ & 8.3 & 9.6 & 50.8 & 59.5 & 37.2 & 28.6 & 3.7 & 2.3 \\
\hline $17 \%$ & 9.2 & 10.3 & 54.6 & 62.7 & 33.4 & 25.3 & 2.8 & 1.7 \\
\hline $22 \%$ & 10.6 & 11.8 & 57.6 & 64.1 & 29.7 & 23.7 & 2.1 & 1.0 \\
\hline
\end{tabular}

From the direction angle distribution (figure 9), the change of pore direction after water immersion in different water contents can be seen. With the increase of water content, the particles are arranged in the direction of order, and the anisotropy shows an increasing trend. Because the loess soil is affected by the combination of force and water, the original soil structure is destroyed, and the large pores existing in the soil will be deformed after being squeezed.

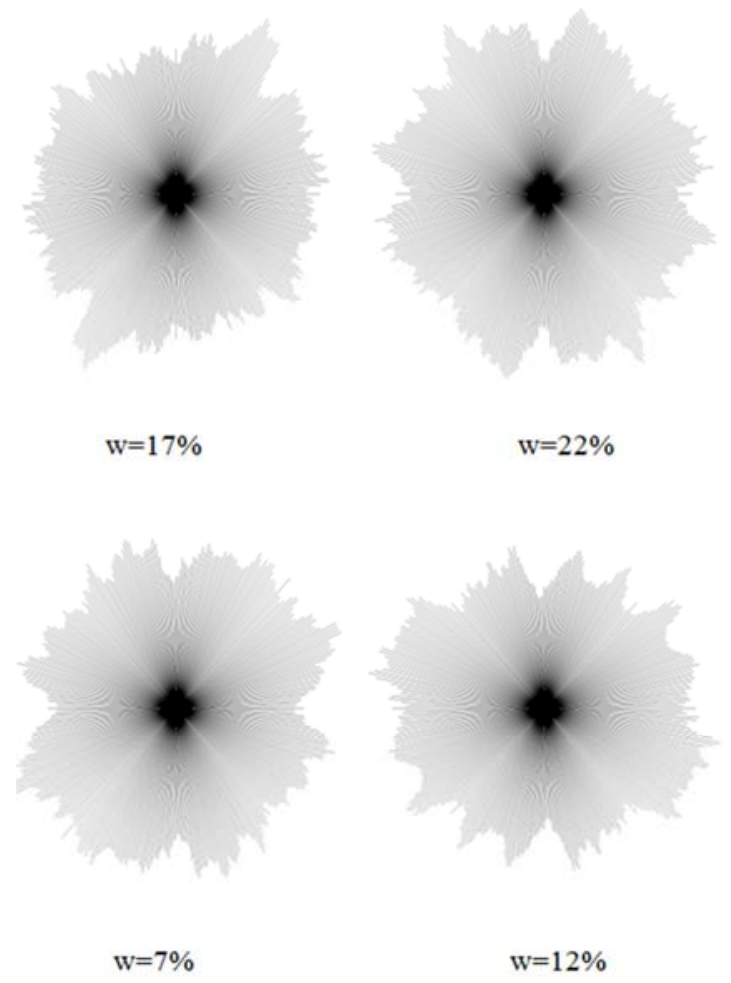

Figure 9. Directional angular distribution of loess with different water content.

The skeleton unit in the soil rotates and rearranges, and the soil directionality of the pore area is relatively weak, so that the area of the surrounding pores and the like are inevitably changed. With the increase of water content, the more uniform the distribution of the pore area of the soil in each angular region after immersion, the greater the disturbance of the soil, and the more the original microstructure of the soil changes.

\section{Conclusion}

(1) Under the same humidified water content, the greater the immersion pressure, the greater the vertical deformation of the sample after immersion in water saturation, and the greater the collapsibility. The lower the humidification water content, the greater the vertical deformation of the sample after immersion in water saturation under the same immersion pressure, and the greater the collapsibility. On the contrary, when the humidification water content is high, the main form of deformation is the compression deformation before immersion. The amount of wet trap will decrease.

(2) With the increase of water content, under the joint action of pressure and water, the soil structure tends to be dense, the particle filling area becomes larger, and the pore filling area decreases. The collapsible process of loess is characterized by the transition from unstable stent contact to stable inlaid contact between the framework particles. A large amount of fine particles are adhered to the surface of the particles. The number of large and medium pores is obviously reduced, the number of small and medium porosity is increased, and the pores are filled.

(3) With the increase of water content, the average pore diameter, average pore area and average pore circumference of the soil gradually decrease after immersion, the content of large and medium pores decreases, the content of micro and small pores increases, and the pore area is decreases. The more uniform the distribution in each angular region, the smaller the porosity of the loess after the collapsing occurs, and the denser grain structure is related to the macroscopic collapsible deformation.

\section{Acknowledgements}

The study was supported by the Qinghai Provincial Department of Science and Technology Applied Basic Research Project (2018-ZJ-749), National Natural Science Foundation of China (51768060).

\section{References}

[1] Z. S. Guo. Analysis of deformation characteristics and stability of loess steep slope under humidification conditions [J]. Chinese Journal of Underground Space and Engineering, 2018, 14 (S1): 445-450+485.

[2] W. Zhang. Research on Humidification Deformation Characteristics of Remolded Loess in Northwest China [J]. Water Resources Planning and Design, 2018 (01): 119-121. 
[3] C. L. Chen, P. Gao, Z. Q. Hu. The Humidification Deformation Characteristics of Loess and Its Relationship with Structural Properties [J]. Chinese Journal of Rock Mechanics and Engineering, 2006 (07): 1352-1360.

[4] S. J. Shao, F. F. Zhou, C. X. Song. Analysis of Foundation Humidification Compression Deformation Considering Structural Changes of Loess [J]. China Civil Engineering Journal, 2006 (06): 94-99.

[5] M. H. Zhang, Y. L. Xie, Liu Health. Characteristics of the collapsibility coefficient curve of loess during the increase (decrease) of wet soil [J]. Rock and Soil Mechanics, 2005 (09): 1363-1368.

[6] Z. S. Guo. Analysis of deformation characteristics and stability of loess steep slope under humidification conditions [J]. Chinese Journal of Underground Space and Engineering, 2018, 14 (S1): 445-450+485.

[7] F. L. Li. Effects of hydraulic water path on mechanical and hydraulic characteristics of loess under confined conditions [D]. Xi'an University of Technology, 2018.

[8] X. X. Shao, H. Y. Zhang, D. J. He, et al. The process of unsaturated humidification deformation of compacted loess and its microscopic mechanism [J]. Journal of Yangtze River Scientific Research Institute, 2019, 36 (04): 82-87+92.

[9] X. X. Shao. Study on unsaturated unsaturated deformation characteristics of loess [D]. Lanzhou University, 2018.
[10] W. Zhang. Research on Humidification Deformation Characteristics of Remolded Loess in Northwest China [J]. Water Resources Planning and Design, 2018 (01): 119-121.

[11] A. J. Zhang, Y. C. Xing, X. L. Hu, et al. Analysis of influencing factors of strong self-weight collapsibility of Yili loess [J]. Chinese Journal of Geotechnical Engineering, 2016, 38 (S2): 117-122.

[12] X. W. Fang, Y. X. Ou, C. H. Li, et al. Study on the influence of wetting on microstructure and mechanical properties of undisturbed $\mathrm{Q}_{2}$ loess. J. Rock and Soil Mechanics, 2015, 36 (S2): 111-117.

[13] X. W. Fang, C. N. Shen, C. H. Li, et al. Remolding the microstructure of $\mathrm{Q}_{2}$ loess. J. Chinese Journal of Underground Space and Engineering, 2014, 10 (06): 1231-1236+1329.

[14] X. W. Fang, C. N. Shen, C. H. Li, et al. Microstructure characteristics and quantitative analysis of loess in Pucheng, Shaanxi Province. J. Chinese Journal of Rock Mechanics and Engineering, 2013, 32 (09): 1917-1925.

[15] X. W. Fang, C. N. Shen, L. Wang, et al. Study on microstructure change of $\mathrm{Q}_{2}$ loess before and after water immersion. J. Rock and Soil Mechanics, 2013, 34 (05): 1319-1324. 